\title{
HUMAN BODY TRACKING FOR DIGITAL ACTORS
}

\author{
Atsushi Nakano $^{\dagger}$, Junichi Hoshino ${ }^{\dagger} \ddagger$ \\ tUniversity of Tsukuba † PRESTO, JST
}

\begin{abstract}
Tracking human body from video is important for many applications such as creating digital actors. However, when the intensity information is partly missing due to the occlusions, the parameter estimation can be easily failed. In this paper, we propose a human motion estimation method by minimizing both intensity change and torque applying to the body parts. The minimum torque criterion adds extra constraints during the motion estimation. This paper presents the theoretical background, and also experimental results that demonstrate the robustness of the tracking.
\end{abstract}

Key words: human body tracking, virtual human, interactive movie

\section{Introduction}

Tracking human body from video is useful for entertainment applications such as creating digital actors and merging graphics with video[8]. If we can estimate $3 \mathrm{D}$ human motion from video, we would be able to use it as a motion capture method without expensive sensors.

The most of conventional method use image features such as gradient, silhouette, color segments, and image contours [1,2,3,4,5,6]. However, when the intensity information is partly missing due to the occlusions, the parameter estimation becomes unstable. The similar problem occurs for silhouette based methods because hands and legs need to be separated from body to obtain best results.

One of the possible solutions for the problem of occlusion may be using multiple views taken by many cameras. However, using many cameras

The original version of this chapter was revised: The copyright line was incorrect. This has been corrected. The Erratum to this chapter is available at DOI: 10.1007/978-0-387-35660-0_65 
requires specialized studio setup that may be too costly for personal applications. In addition, it is still difficult to capture the whole appearance when the multiple people exists within the same camera view.

In this paper, we propose a human motion estimation method by minimizing both intensity difference and joint torque along the long action trajectory. The minimum joint torque criterion adds extra constraints during the motion estimation. We optimize the whole motion trajectory along the long image sequences to increase the robustness of the estimation.

This paper presents the theoretical background, and also experimental results that demonstrate the robustness of the tracking. We show that the complex, dynamic human action such as dance sequences can be captured from video.

\section{Overview}

Capturing actor's motion and interaction from video is useful for creating interactive movies because we do not need expensive studios for motion capture(Fig.1). The existing movie or television footages can be also used for learning actor's behaviours.
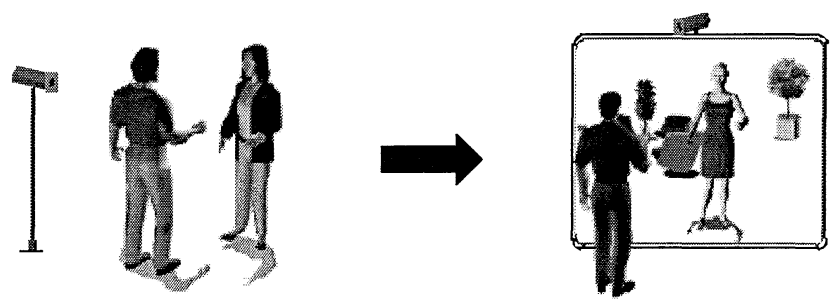

Figure 1: Capturing actor's motion and interactions for interactive movies

We increase the robustness of the tracking by using the long image sequences, and minimizing joint torques along the motion trajectory. We represent the continuous angler motion in a few parameters using the hermite interpolation to decrease the degree of freedom. The parameter reduction also helps the faster convergence and the increases in the stability. The body models are approximated by a polyhedron made by CAD modeler. The model has a tree structure with a root at the trunk, and has a local coordinate system whose origin is located at a joint. The dynamic model of human body can be derived from Newton-Euler method. 
In the following sections, we explain the detail of the dynamic modeling of the human body, and the optimization of joint torque along the motion trajectory.

\section{Dynamic Modeling of Human Body}

Newton-Euler formulation desribes the motion of the links in terms of a balance of forces and moments acting on it [7,8]. Consider the generic augmented link of the human body kinematic chain. Newton equations for the translational motion of the center of mass can be written as

$$
\boldsymbol{F}=\mathrm{m} \dot{\boldsymbol{v}}
$$

The Eular equations for the rotational motion of the link (referring moments to the center of mass can be written as

$$
\boldsymbol{N}=\boldsymbol{I} \boldsymbol{\omega}+\boldsymbol{\omega} \times(\boldsymbol{I} \boldsymbol{\omega})
$$

where

F : force exerted on link

$v$ : linear velocity of center of mass

$I$ : inertia tensor of augmented link $m:$ is a mass of augmented link

$\mathbf{N}$ : moment of inertial of rotator $\omega$ : angular velocity of link

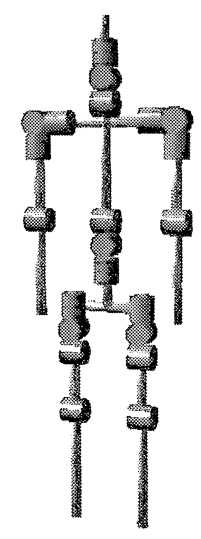

Figure 2: Human joint model

Once the joint positions, velocities and accelerations are known, one can compute the link velocities and accelerations, and the Newton-Euler equations can be utilized to find the forces and moments acting on each link in a recursive fashion, starting from the force and moment applied to the end 
effectors. On the other hand, also link and rotor velocities and acceleration can be computed recursively starting from the velocity and acceleration of the base link. In sum, a computationally recursive algorithm can be constructed that features a forward recursion relative to the propagation of velocities and accelerations and a backward recursion for the propagation of forces and moments along the structure.

Let the human body has $s$ branch, and branch $k$ has $n_{k}(k=1, \ldots, s)$ links. The calculation of the joint torque can be done in the following steps.

1) The acceleration, angular velocity, angular accelerations of blanch $1,0^{\text {th }}$ link can be specified as

$$
\dot{\boldsymbol{v}}_{0}^{1}=-\boldsymbol{g}, \quad \boldsymbol{\omega}_{0}^{1}=0, \quad \dot{\boldsymbol{\omega}}_{0}^{1}=0
$$

2) Compute $\dot{\boldsymbol{v}}_{i}^{1}, \quad \boldsymbol{\omega}_{i}^{1}, \quad \dot{\boldsymbol{\omega}}_{i}^{1}$ from $i=1$ to $n_{1}$ for branch1.

3) The initial value of the angular velocity and angular accelerations of branch $k(k=2, \ldots, s)$ can be obtained as

$$
\begin{aligned}
& \dot{\boldsymbol{v}}_{0}^{k}=\left(\boldsymbol{B}_{R}^{k}\right)^{T}\left\{\dot{\boldsymbol{v}}_{c o l}^{c o b}+\dot{\boldsymbol{\omega}}_{c o l}^{c o b} \times \widetilde{\boldsymbol{B}}_{p}^{k}+\boldsymbol{\omega}_{c o l}^{c o b} \times\left(\boldsymbol{\omega}_{c o l}^{c o b} \times \widetilde{\boldsymbol{B}}_{p}^{k}\right)\right\} \\
& \boldsymbol{\omega}_{0}^{k}=\left(\boldsymbol{B}_{R}^{k}\right)^{T} \boldsymbol{\omega}_{c o l}^{c o b}, \quad \dot{\boldsymbol{\omega}}_{0}^{k}=\left(\boldsymbol{B}_{R}^{k}\right)^{T} \dot{\boldsymbol{\omega}}_{c o l}^{c o b}
\end{aligned}
$$

where $\mathbf{B}^{k}$ is a $4 \times 4$ coordinate transform matrix from a parent branch to a child branch in a homogeneous coordinate system. $\mathbf{B}_{R}^{k}$ shows the left-above $3 \times 3$ matrix of $\mathbf{B}^{k}$, and $\overrightarrow{\mathbf{B}}_{p}^{k}$ is a right-above matrix of $\left(\mathbf{B}^{k}\right)^{-1}$. cob is a branch number of branch $k$, and col is a link number of branch $k$.

4) Calculate $\dot{\boldsymbol{v}}_{i}^{k}, \quad \boldsymbol{\omega}_{i}^{k}, \quad \dot{\boldsymbol{\omega}}_{i}^{k}$ for branch $k(2, \ldots, s)$ from $i=1$ to $n_{k}$

5) Calculate force $\boldsymbol{f}_{\mathrm{i}}{ }^{\mathrm{k}}$ and moment $\mathbf{n}_{i}^{k}$ for blanch $k(s, \ldots, 1)$ from $i=n_{k}$ to 1 .

The joint torque $\boldsymbol{\tau}_{i}^{k}$ can be estimated as follows

Revolute joint: $\boldsymbol{\tau}_{\mathrm{i}}^{\mathrm{k}}=\boldsymbol{z}_{0}^{\mathrm{T}} \boldsymbol{n}_{\mathrm{i}}^{\mathrm{k}}$

Slide joint: $\quad \boldsymbol{\tau}_{\mathrm{i}}^{\mathrm{k}}=\boldsymbol{z}_{0}^{\mathrm{T}} \boldsymbol{f}_{\mathrm{i}}^{\mathrm{k}}$

where $\boldsymbol{z}_{0}=\left[\begin{array}{lll}0 & 0 & 1\end{array}\right]^{T}$

The equation of the human motion dynamics can be represented as follows:

$$
\tau=M(q) \ddot{q}+h(q, \dot{q})+g(q)
$$


where $\boldsymbol{M}(\boldsymbol{q})$ is inertia tensor matrix, $\boldsymbol{h}(\boldsymbol{q}, \dot{\boldsymbol{q}})$ represent centrifugal and Corioli's force, $\boldsymbol{g}(\boldsymbol{q})$ is a gravity term, $\boldsymbol{q}$ is a joint angle.

Right side of eq. (9) consists of constant values except angler velocity and angler accelerations. Therefore we can obtain the joint torques by specifying them as described in Sec. 4.2

\section{Optimizing Motion Trajectory}

In this section, we show the motion tracking method using the minimum torque criterion. We optimize the joint torques along the whole motion trajectory, and also the least squares difference of image intensity.

\subsection{Initial estimate of motion trajectory}

First we obtain initial estimate of motion trajectory by using the sparsely estimated 3D pose and the simple interpolation technique. We use the silhouette-based pose alignment algorithm[8] for the initial estimation. This type of pose estimation technique is only useful when the hands and legs are clearly separated from the body. Therefore we still need the global optimization technique to estimate continuous human motion at every frames.

We use the curve fitting by the piecewise hermite interpolation using the $3^{\text {rd }}$ order bezier-spline. We need to specify the sample point and tangent vectors to estimate the curve. Let the node of $n+1$ be $\mathbf{Q}_{0}, \mathbf{Q}_{1}, \ldots, \mathbf{Q}_{n}$, we can obtain the tangent vectors by

$$
\mathbf{I}_{0}=\frac{\mathbf{Q}_{1}-\mathbf{Q}_{0}}{\left|\mathbf{Q}_{1}-\mathbf{Q}_{0}\right|}, \mathbf{I}_{n}=\frac{\mathbf{Q}_{n}-\mathbf{Q}_{n-1}}{\left|\mathbf{Q}_{n}-\mathbf{Q}_{n-1}\right|}, \quad \mathbf{I}_{i}=\frac{\mathbf{Q}_{i}-\mathbf{Q}_{i-1}}{\left|\mathbf{Q}_{i}-\mathbf{Q}_{i-1}\right|}
$$

Then we obtain the interpolated curve $\{P(t) \mid 0 \leq t \leq n\}$ where $t$ is a frame number. We use $P(t)$ as an initial value for the pose estimate. Such parametric representation also has the advantage of decreasing the degree of freedom to stabilize the estimation.

\subsection{Obtaining angular velocity and acceleration}

To estimate joint torque using eq. (9), we need an angular velocity and angular acceleration of the joint angle. The velocity $\dot{\boldsymbol{q}}_{\mathrm{f}}$ and the acceleration $\ddot{\boldsymbol{q}}_{\mathrm{f}}$ of $\mathrm{f}$ th frame are obtained by 


$$
\begin{gathered}
\dot{\boldsymbol{q}}_{\mathrm{f}}=\frac{\frac{\boldsymbol{q}_{\mathrm{f}+1}-\boldsymbol{q}_{\mathrm{f}}}{\mathrm{H}}+\frac{\boldsymbol{q}_{\mathrm{f}}-\boldsymbol{q}_{\mathrm{f}-1}}{\mathrm{H}}}{2}=\frac{\boldsymbol{q}_{\mathrm{f}+1}-\boldsymbol{q}_{\mathrm{f}-1}}{2 \mathrm{H}} \\
\ddot{\boldsymbol{q}}_{\mathrm{f}}=\frac{\frac{\boldsymbol{q}_{\mathrm{f}+1}-\boldsymbol{q}_{\mathrm{f}}}{\mathrm{H}}-\frac{\boldsymbol{q}_{\mathrm{f}}-\boldsymbol{q}_{\mathrm{f}-1}}{\mathrm{H}}}{\mathrm{H}}=\frac{\boldsymbol{q}_{\mathrm{f}+1}-2 \boldsymbol{q}_{\mathrm{f}}+\boldsymbol{q}_{\mathrm{f}-1}}{\mathrm{H}^{2}}
\end{gathered}
$$

where $\mathrm{H}$ : frame time interval, $\mathrm{f}:$ frame number, fMax: total frame number, $\boldsymbol{q}_{\mathrm{f}}$ : joint angle of $\mathrm{f}$ th frame

\subsection{Evaluation function and optimization}

The total of joint torques $\boldsymbol{T}_{k}$ at $\mathrm{k}$ th link Link_k can be calculated using the torque value $\tau_{f}$ at $\mathrm{f} t h$ frames:

$$
\boldsymbol{T}_{k}=\sum_{f=1}^{f M a x-2} \boldsymbol{\tau}_{f}
$$

$\mathrm{f}$ is from 1 to $f M a x-2$ because the velocity and acceleration can be only estimated within these frames from eq. (11), (12). The resulting $\boldsymbol{\tau}_{f}$ has the same range.

The total of the intensity value $\mathbf{D}_{k}$ of Link_k can be obtained as

$$
\boldsymbol{D}_{k}=\sum_{f=1}^{f M a x-2}\left(i_{f}-i_{f-1}\right)^{2}
$$

where $i_{f}$ is the total of intensity valued of each area corresponding to the body parts. The occlusion by the body parts can be judged by using the $3 \mathrm{~d}$ body model and z-buffer.

By using eq.(13), (14), the evaluation function can be written as

$$
\alpha_{k} \boldsymbol{T}_{k}+\beta_{k} \boldsymbol{D}_{k} \rightarrow \min
$$

where $\alpha_{k}, \beta_{k}$ is a weight functions. We use the greedy method to optimize eq. (15). By perturbing the control points of spline curve around the small 
neighborhoods, we find the motion trajectory which minimize eq.(15). In our experiments, this simple optimization algorithm works well for complex actions.

\section{Experiments}

We have implemented our algorithm using standard PC to test the effectiveness of the tracking. Fig. 3 shows the result of tracking using the video sequence including complex and dynamic behavior.

\section{Conclusions}

We proposed a human motion estimation method by minimizing both intensity change and torque applying to the body parts. The minimum torque criterion adds extra constraints during the motion estimation. We also demonstrated the performance of our algorithm using the complex behaviours such as dance sequences. In future, we extend our algorithm to estimate articulated and non-rigid motion such as animals.

\section{References}

[1] C. Bregler and J. Malik. Tracking people with twists and exponential maps. Proc. IEEE Comput. Soc. Conf. Comput. Vision and Pattern Recogn., pages 8-15, 1998.

[2] D. Gavrila and L. Davis. 3d model-based tracking of humans in action: A multi-view approach. Proc. IEEE Comput. Soc. Conf. Comput. Vision and Pattern Recogn., pages 7380, 1996.

[3] I. Kakadiaris and D. Metaxas. Model-based estimation of 3d human motion. IEEE Trans. PAMI, 22(12):1453-1459, 2000.

[4] J. M. Rehg and T. Kanade. Visual tracking of high DOF articulated structures: An application to human hand tracking. Lecture Notes in Computer Science, 800:35-46, 1994.

[5] C. Wren, A. Azarbayejani, T. Darrell, and A. Pentland. Pfinder: Real-time tracking of the human body. IEEE Trans. PAMI, 19(7):780-785, July 1997.

[6] M. Yamamoto and K. Koshikawa. Human motion analysis based on a robot arm model. Proc. IEEE Comput. Soc. Conf. Comput. Vision and Pattern Recogn., pages 664-665, 1991.

[7] H. Kawasaki and T. Shimizu, "Development of Robot Symbolic Analysis System ROSAM II," Proc. of Int. Conf. Of Japan-USA Symposium on Flexible Automation, Ohtu, Japan, pp.1121-1128, 1998.

[8] J. Y. S. Luh, M. W. Walker, and R. P. C. Paul, "On-Line Computational Scheme for Mechanical Manipulators", IEEE Int.Jour. of Dynamic Systems, Measurement, and Control, No. 102, pp. 69-76,1980

[9] Jun'ichi Hoshino, Hirofumi Saito:" A Match Moving Technique for Human Body Sequences” , ICASSSP2001, Conference Proceedings CD-ROM, IMDSP-P3.1, 2001 

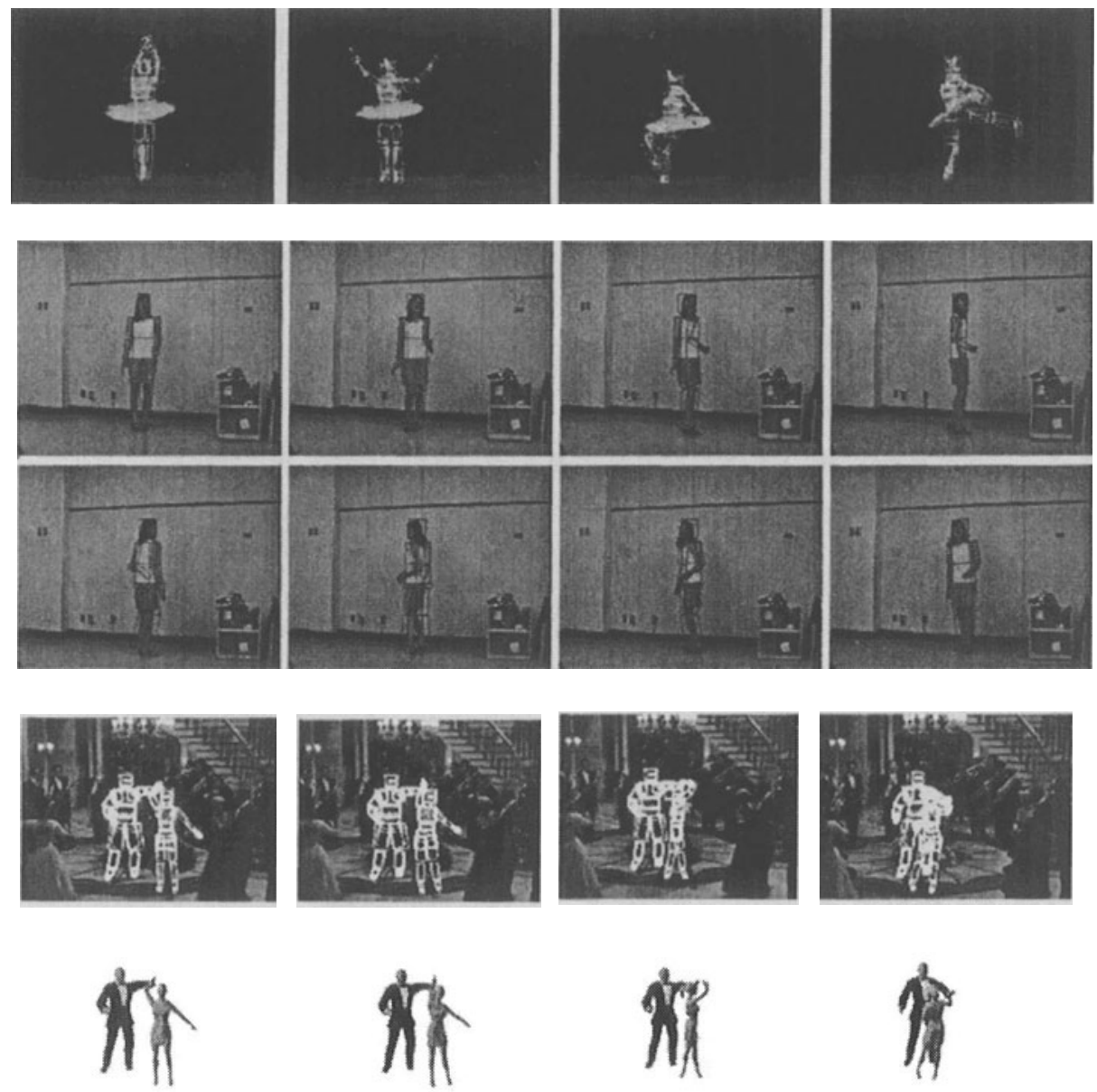

Figure 3: Results of motion capture from video. 